\title{
Systemic Lymphadenopathy as the Initial Presentation of Malignant Mesothelioma: A Report of Three Cases
}

\author{
Yaxia Zhang, Zohreh M. Taheri, and Merce Jorda \\ Department of Pathology, University of Miami-Miller School of Medicine/Jackson Memorial Hospital, \\ Miami, FL 33136, USA \\ Correspondence should be addressed to Merce Jorda, mjorda@med.miami.edu
}

Received 5 August 2009; Accepted 19 October 2009

Academic Editor: Piero Tosi

Copyright (๑) 2010 Yaxia Zhang et al. This is an open access article distributed under the Creative Commons Attribution License, which permits unrestricted use, distribution, and reproduction in any medium, provided the original work is properly cited.

Systemic lymph node metastasis is a rare event in malignant mesothelioma. It is even more exceptional when systemic lymph node metastasis is the initial clinical presentation. Review of literature discloses only four cases in which metastatic lymphadenopathy was the only symptom of malignant mesothelioma. We, herewith, report three cases where the initial diagnosis of malignant mesothelioma was made by biopsy of enlarged lymph nodes, which were the only clinical presentation. Immunohistochemistry played a pivotal role in elucidating the mesothelial origin of their unusual systemic lymph node metastasis.

\section{Introduction}

Malignant mesothelioma (MM) is an uncommon neoplasm which is characterized by highly aggressive behavior and poor prognosis [1]. The neoplasm predominantly involves pleural and peritoneal cavities, with a smaller percentage of cases arising in the pericardial sac and testicular tunica vaginalis [2]. Clinically, the majority of patients have local symptoms such as chest or abdominal pain and dyspnea, depending on the site of origin. Occasionally, patients may present with distant metastasis. Systemic lymphadenopathy, however, is an exceeding rare initial presentation of this disease [3]; thirteen cases have been reported in literature. In four of those, systemic lymphadenopathy was the only clinical manifestation [4-6].

In this report, we describe three additional cases of primary peritoneal MM in which the initial diagnosis of the disease was made by biopsy of neck, supraclavicular lymph nodes, and axillary lymph nodes. Two cases were primary from peritoneum and the third originated in the pleura.

\section{Report of Cases}

2.1. Case One. A 50-year-old male with no past medical history presented with progressive enlargement of lymph nodes in his left groin and right inferior neck over a period of 6 months. PET scan showed high uptake in several areas such as mediastinum, pericardial region, cardiophrenic angles, supraclavicular area, internal mammary, perihepatic region, and groin with standardized uptake values (SUVs) ranging from 4.8 to 12.5 . Most of these uptakes were interpreted as presence of a malignant process probably malignant lymphoma. Immediate assessment on fine needle aspiration cytology from left groin lymph node, however, ruled out the diagnosis of malignant lymphoma. A subsequent core biopsy was obtained from right supraclavicular node and was interpreted as metastatic malignant epithelial neoplasm to lymph node. The neoplasm was characterized by a diffuse growth of polygonal cells with well-defined cell membranes and dense eosinophilic cytoplasm. Nuclei were generally single, and mitoses were scant (Figure 1). Tumor cells were positive for cytokeratin (Figure 2), calretinin (Figure 3), D240 (Figure 4), CD10, and CK5/6, focally positive for CK7 and CA-125, and negative for hepatocellular antigen, renal carcinoma antigen, prostatic specific antigen, carcinoembryonic antigen, HMB45, S100 protein, p63, CDX2, CK20, thyroid transcription factor-1, inhibin, and alpha-feto protein by immunohistochemistry. Based on histomorpholgy and immunophenotype, the diagnosis of metastatic malignant mesothelioma was rendered. Retrospectively, review of 


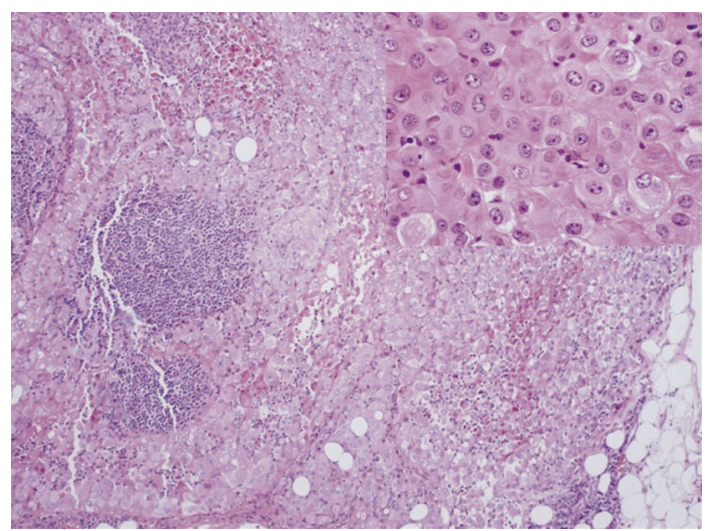

Figure 1: Metastatic malignant mesothelioma to lymph node. $(\mathrm{H} \& \mathrm{E}, 10 \times)$. The inset shows that the tumor cells are polygonal with well-defined cell membranes and dense eosinophilic cytoplasm (H\&E, 60×).

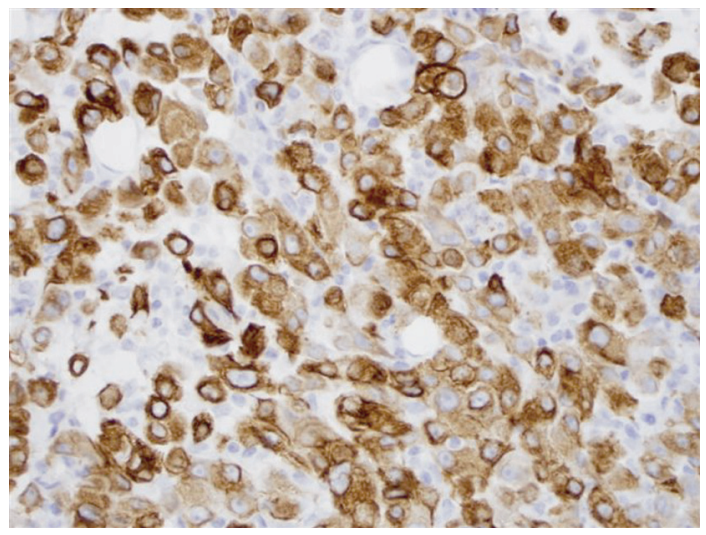

FIGURE 2: Metastatic malignant mesothelioma to lymph node. The tumor cells are positive for cytokeratin by immunohistochemistry (Keratin, $40 \times$ ).

clinical and imaging studies confirmed that the neoplasm arised from peritoneum in perihepatic region.

2.2. Case Two. A 61-year-old male with no past medical history presented with left neck lymphadenopathy. CT scan showed multiple masses in the neck region, mediastinum and abdomen. Biopsy from neck mass revealed several matted lymph nodes with complete replacement by a nonlymphoid malignant neoplasm. The cells were arranged in organoid and trabecular pattern with areas of necrosis with perinodal tumoral involvement. The tumor cells were large and polygonal with eosinophilic cytoplasm, large prominent nucleoli, and numerous mitoses (Figure 5). Tumor cells were positive for cytokeratin and calretinin by immunohistochemistry. They were negative for CD20, CD45, CD3, S-100 protein, thyroglobulin, carcinoembryonic antigen, human chorionic gonadotropin, keratin $8 / 18$, thyroid transcription factor-1, inhibin, and renal carcinoma antigen. A diagnosis of metastatic malignant mesothelioma

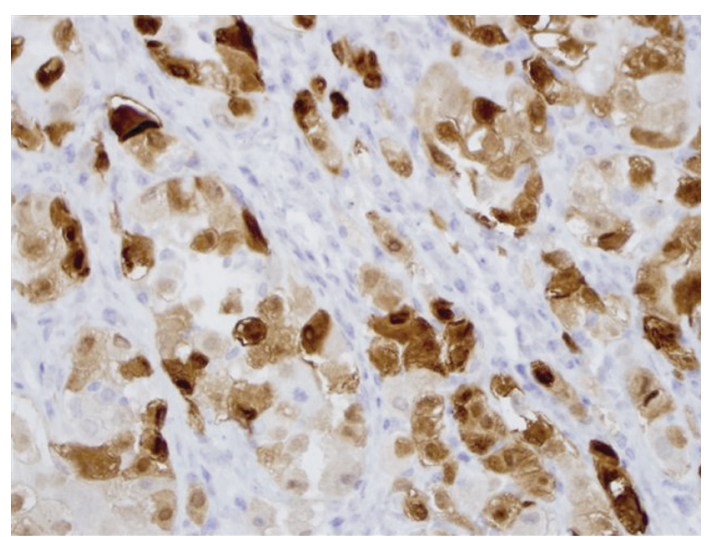

Figure 3: Metastatic malignant mesothelioma to lymph node. The tumor cells are positive for calretinin by immunohistochemistry (Calretinin, $40 \times$ ).

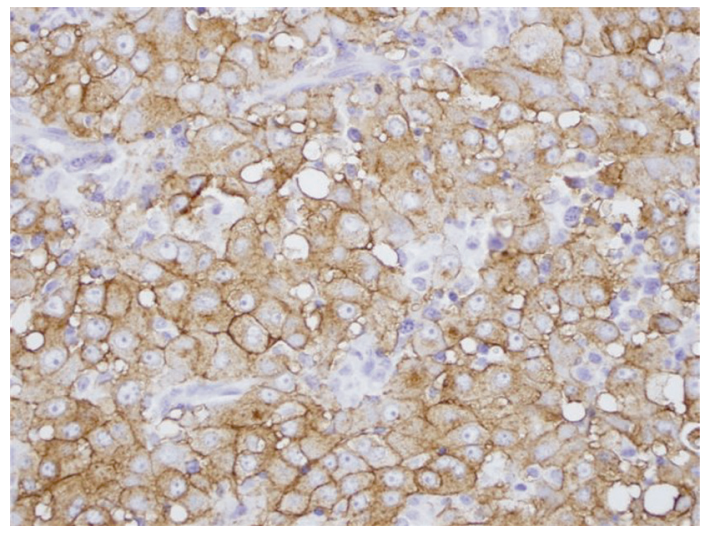

FIGURE 4: Metastatic malignant mesothelioma to lymph node. The tumor cells are positive for D2-40 by immunohistochemistry (D240, 40×).

was made. Retrospectively, review of prior CT scans revealed that the neoplasm originated in pelvic peritoneum.

2.3. Case Three. Patient is a 69-year-old male who by imaging proved to have mediastinal and left axillary lymphadenopathy. Biopsy of the axillary lymph node showed a metastatic epithelial neoplasm morphologically consistent with malignant mesothelioma. Positive immunohistochemical reaction for calretinin and negative staining for TTF-1 and CEA supported that diagnosis. Clinical and imaging studies revealed left pleural thickening. A pleural biopsy confirmed the diagnosis of epithelioid malignant mesothelioma.

\section{Comment}

Malignant mesothelioma is characterized by an aggressive local behavior, such as pain and accumulation of fluid in the region of origin [7]. Rarely, this neoplasm metastasizes 


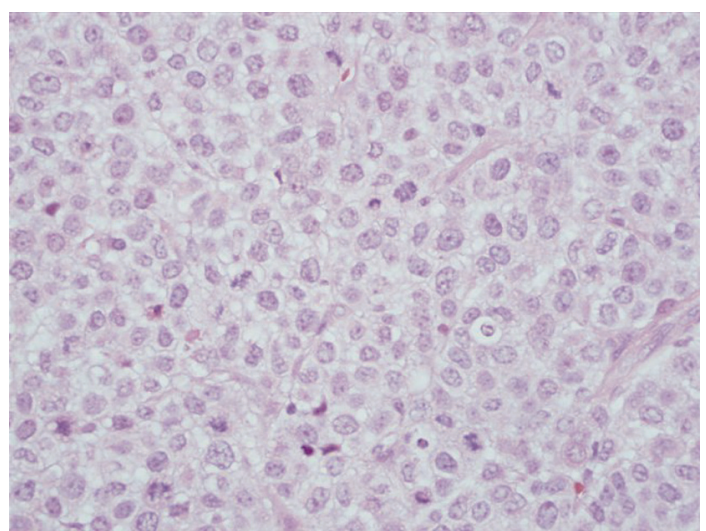

Figure 5: Metastatic malignant mesothelioma to lymph node. The tumor cells are large and polygonal with eosinophilic cytoplasm. They have prominent nucleoli and display numerous mitoses $(\mathrm{H} \& \mathrm{E}$, $40 \times)$.

to lymph nodes, particularly early in the course of disease. In the majority of cases, metastatic spread to the lymph nodes is detected simultaneously with other conventional clinical features of malignant mesothelioma, such as pain and pleural or peritoneal effusions. Malignant mesothelioma presenting with peripheral lymphadenopathy as the initial symptom is exceptionally rare. To our knowledge, there have been 13 cases of MM with clinically apparent and histopathologically proven lymph node metastasis [4-13] but in only 4 of them, metastatic lymphadenopathy was the initial clinical presentation. Two of these cases were originated in peritoneum [4], each from pleura [6], and pericardium [5]. In our case one, PET scan showed numerous systemic enlarged lymph nodes, with the largest lymph node present at the mediastinum. Retrospectively, the area with high standardized uptake values present at the perihepatic region was considered to be the primary site of the neoplasm. Similarly, in our case two, retrospective analysis of imaging studies revealed the site of origin. In our case three, the biopsy from pleural surface confirmed the diagnosis of MM which was initially made by sampling patient's axillary lymph node.

When one confronts a metastatic epithelial neoplasm to a lymph node with evidence of a primary site, a malignant mesothelioma is usually not on the list of potential differential diagnoses. A combination of histomorphology along with an appropriate immunohistochemical tests, however, may elucidate the mesothelial origin of such rare metastasis. Immunohistochemistry is the most helpful ancillary test in confirmation of the diagnosis, particularly in separating MM from adenocarcinoma. Most useful markers in our experience from $\mathrm{MM}$ are calretinin and D2-40 and negative reaction for CEA, MOC-31, and TTF-1 $[14,15]$.

\section{Acknowledgment}

The authors gratefully acknowledge Dr. M. Nadji for his critical review of the manuscript.

\section{References}

[1] J. Brenner, P. P. Sordillo, G. B. Magill, and R. B. Golbey, "Malignant mesothelioma of the pleura. Review of 123 patients," Cancer, vol. 49, no. 11, pp. 2431-2435, 1982.

[2] J. Jaffe, J. A. Roth, and H. Carter, "Malignant papillary mesothelioma of tunica vaginalis testis," Urology, vol. 11, no. 6, pp. 647-650, 1978.

[3] J. Sussman and J. Rosai, "Mesothelioma metastasis," American Journal of Surgical Pathology, vol. 15, no. 10, pp. 1016-1017, 1991.

[4] J. Sussman and J. Rosai, "Lymph node metastasis as the initial manifastation of malignant mesothelioma. Report of six cases," American Journal of Surgical Pathology, vol. 14, no. 9, pp. 819-828, 1990.

[5] E. Yakirevich, Y. Sova, K. Drumea, I. Bergman, M. Quitt, and M. B. Resnick, "Peripheral lymphadenopathy as the initial manifestation of pericardial mesothelioma: a case report," International Journal of Surgical Pathology, vol. 12, no. 4, pp. 403-405, 2004.

[6] E. J. Wills, "Pleural mesothelioma with initial presentation as cervical lymphadenopathy," Ultrastructural Pathology, vol. 19, no. 5, pp. 389-394, 1995.

[7] K. H. Antman, "Natural history and staging of malignant mesothelioma," Chest, vol. 96, supplement 1, pp. 93S-95S, 1989.

[8] S. B. Kim, B. Varkey, and H. Choi, "Diagnosis of malignant pleural mesothelioma by axillary lymph node biopsy," Chest, vol. 91, no. 2, pp. 279-281, 1987.

[9] N. A. Ansari and N. W. Derias, "Diagnosis of malignant mesothelioma by fine needle aspiration of a cervical lymph node: a case report," Acta Cytologica, vol. 44, no. 1, pp. 70-74, 2000.

[10] M. Huncharek and D. Adcock, "Diagnosis of malignant pleural mesothelioma by supraclavicular lymph node biopsy," Oncology Reports, vol. 6, no. 4, pp. 833-834, 1999.

[11] F. E. Craig, N. F. Fishback, J. G. Schwartz, and C. N. Powers, "Occult metastatic mesothelioma-diagnosis by fineneedle aspiration: a case report," American Journal of Clinical Pathology, vol. 97, no. 4, pp. 493-497, 1992.

[12] R. E. Scully, E. J. Mark, W. F. McNeely, S. H. Ebeling, and L. D. Phillips, "Case records of the Massachusetts General Hospital. Weekly clinicopathological exercises. Case 20-1997. A 74-year-old man with progressive cough, dyspnea, and pleural thickening," The New England Journal of Medicine, vol. 336, no. 26, pp. 1895-1903, 1997.

[13] J. Lloreta and S. Serrano, "Pleural mesothelioma presenting as an axillary lymph node metastasis with anemone cell appearance," Ultrastructural Pathology, vol. 18, no. 1-2, pp. 293-298, 1994.

[14] N. G. Ordóñez, "What are the current best immunohistochemical markers for the diagnosis of epithelioid mesothelioma? A review and update," Human Pathology, vol. 38, no. 1, pp. 1-16, 2007.

[15] N. G. Ordóñez, "Value of immunohistochemistry in distinguishing peritoneal mesothelioma from serous carcinoma of the ovary and peritoneum: a review and update," Advances in Anatomic Pathology, vol. 13, no. 1, pp. 16-25, 2006. 


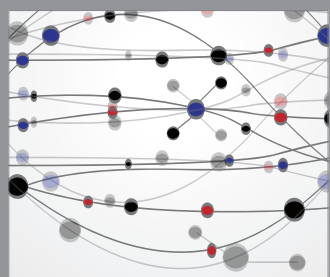

The Scientific World Journal
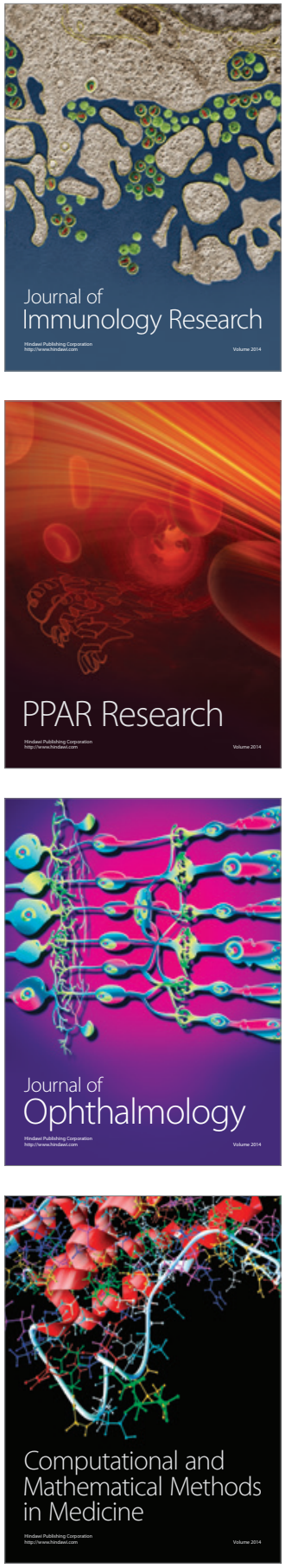

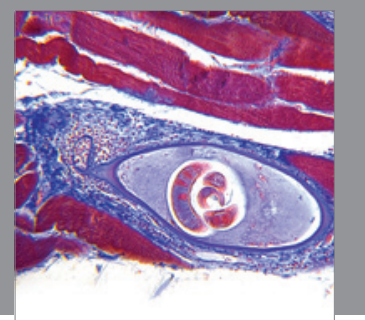

Gastroenterology

Research and Practice
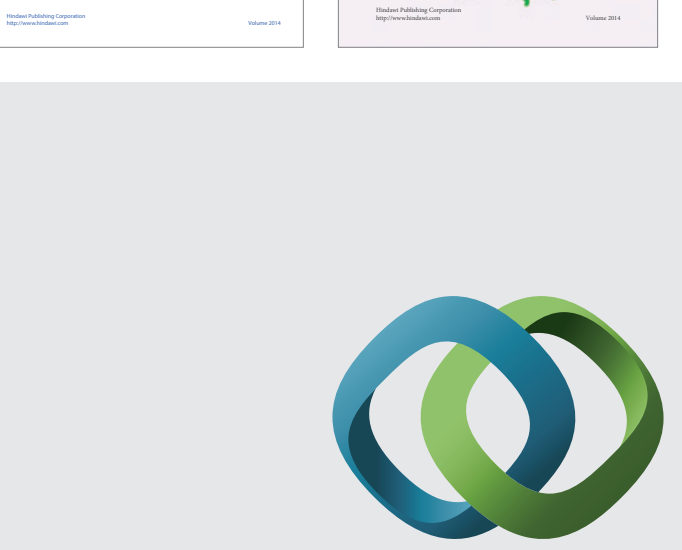

\section{Hindawi}

Submit your manuscripts at

http://www.hindawi.com
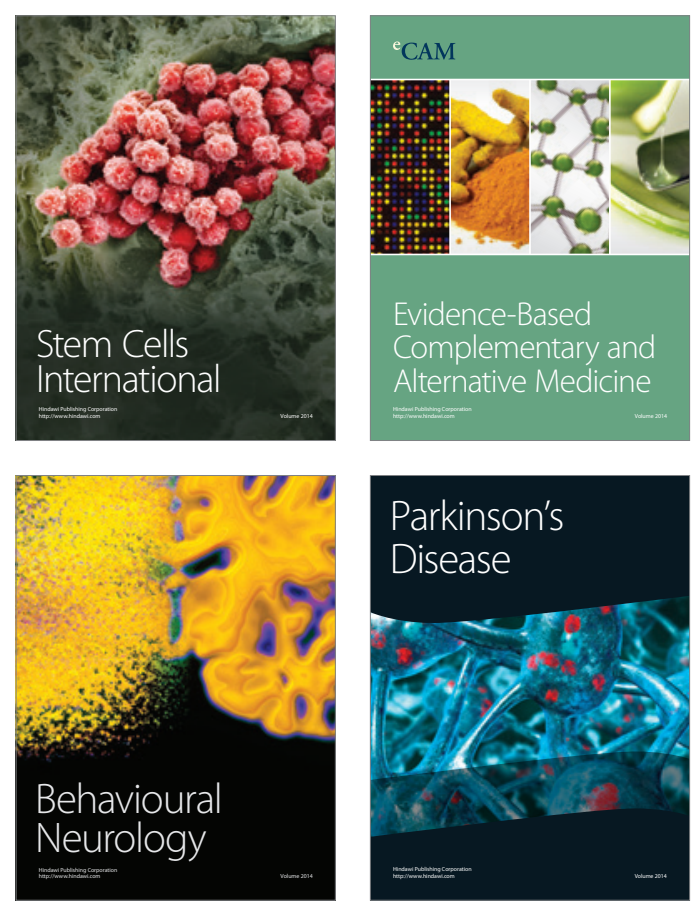

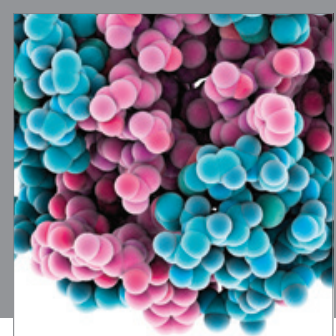

Journal of
Diabetes Research

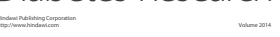

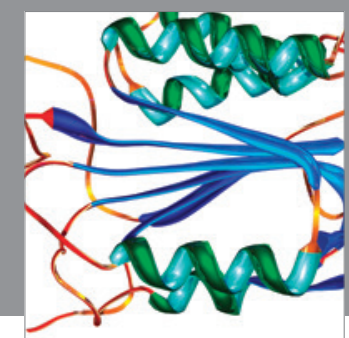

Disease Markers
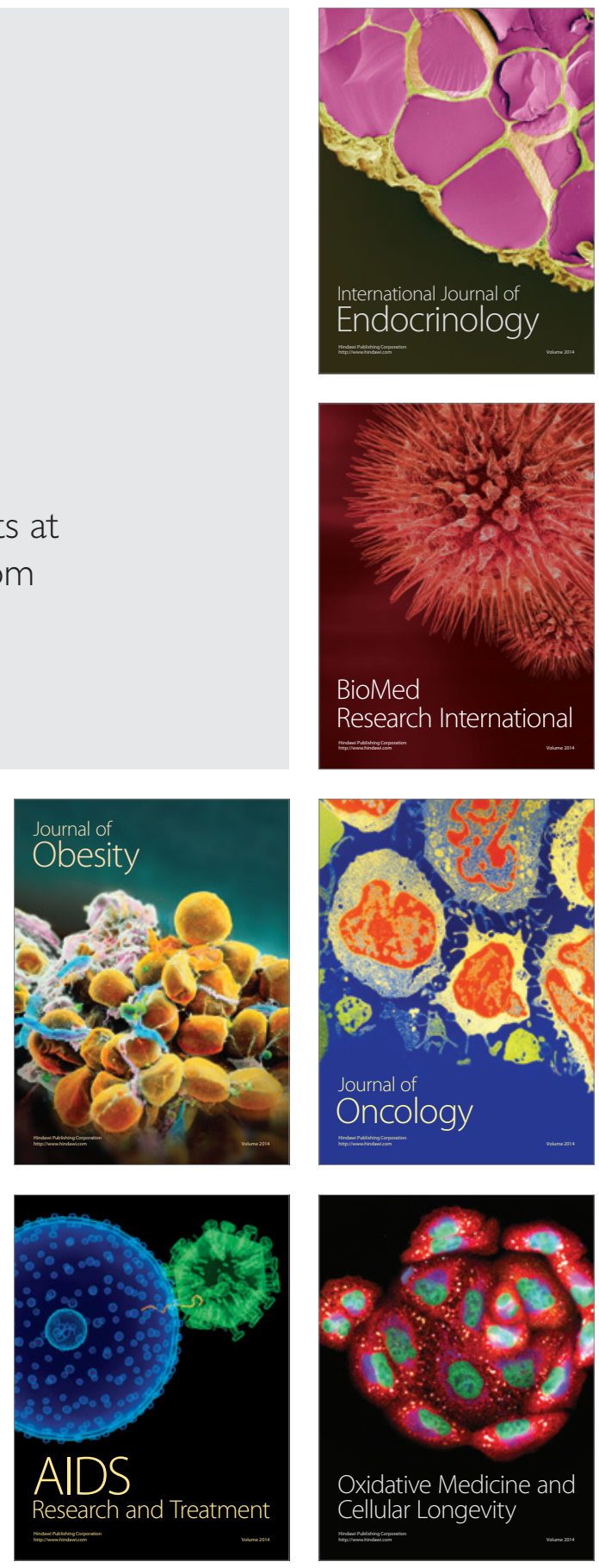